\title{
Joint Signaling Techniques and Spectral Optimization for Symmetric Bit-Rate Communication Over Self-NEXT-Dominated Channels
}

\author{
Rohit V. Gaikwad and Richard G. Baraniuk
}

\begin{abstract}
We present a framework for maximizing the capacity of symmetric bit-rate communication services dominated by Gaussian crosstalk, in particular, digital subscriber line (DSL) services. We solve for optimal transmit power spectral densities (PSDs) that maximize the joint capacity of same-service users and yield significant gains in bit rates (or performance margins) over current schemes. Our results differ from previous work in that we develop transmit spectra in the presence of self-far-end crosstalk in addition to self-near-end crosstalk, present optimal contiguous spectra for practical modulation schemes, and derive optimal spectra under an additional frequency-domain peak-power constraint. Furthermore, by design, the optimal transmit PSDs are spectrally compatible with existing services on neighboring lines.
\end{abstract}

Index Terms-Crosstalk, digital subscriber line (DSL), information rates, multiuser communication, spectral compatibility.

\section{INTRODUCTION}

$\mathbf{I}$ $\mathrm{N}$ THIS LETTER, we derive optimal transmit power spectral densities (PSDs) that maximize the bit rates of symmetric bit-rate communication services dominated by Gaussian crosstalk. While our results are general, we will phrase them in the context of digital subscriber line (DSL) communication to be concrete. In a DSL scenario, a service $\mathcal{S}$ faces self-far-end crosstalk (self-FEXT), self-near-end crosstalk (self-NEXT), and additive Gaussian noise (AGN). We also derive practical contiguous PSDs and transmit PSDs in the presence of an additional frequency-domain peak-power constraint.

We assume the usual DSL environment setup [1] with standard crosstalk, channel, and noise assumptions [3] (see [2], [4], and [5] for detailed introductions to DSL and crosstalk). We divide the transmission bandwidth $B$ of the channel into $K$ narrow frequency bins, each of width $W$ Hertz and center frequencies $f_{k}, k \in\{1, \ldots, K\}$ (see Fig. 1). Let $H_{k}, X_{k}$, and $F_{k}$ denote the magnitude squared of the channel, self-NEXT, and self-FEXT transfer functions in bin $k$. Further, let $N_{k}=N\left(f_{k}\right)$ denote the combined AGN from different service interference and channel noise [6], and $P_{k}$ the power in bin $k$.

Let $S^{u}(f)$ and $S^{d}(f)$ denote the upstream and downstream transmit PSDs, and let $s^{u}(f)$ and $s^{d}(f)$ denote the same in a

Paper approved by C.-L. Wang, the Editor for Wireless Spread Spectrum of the IEEE Communications Society. Manuscript received January 14, 2000; revised October 20, 2000 and April 2, 2001. This work was supported in part by the National Science Foundation under Grant MIP-9457438 and Grant CCR9973188, in part by Nortel Networks, in part by SBC Communications, and the in part by the Texas Instruments Leadership University Program.

R. V. Gaikwad is with Broadcom Corporation, San Diego, CA 92128 USA (e-mail: rgaikwad@broadcom.com).

R. G. Baraniuk is with the Department of Electrical and Computer Engineering, Rice University, Houston, TX 77005 USA (e-mail: richb@rice.edu).

Digital Object Identifier 10.1109/TCOMM.2004.831373

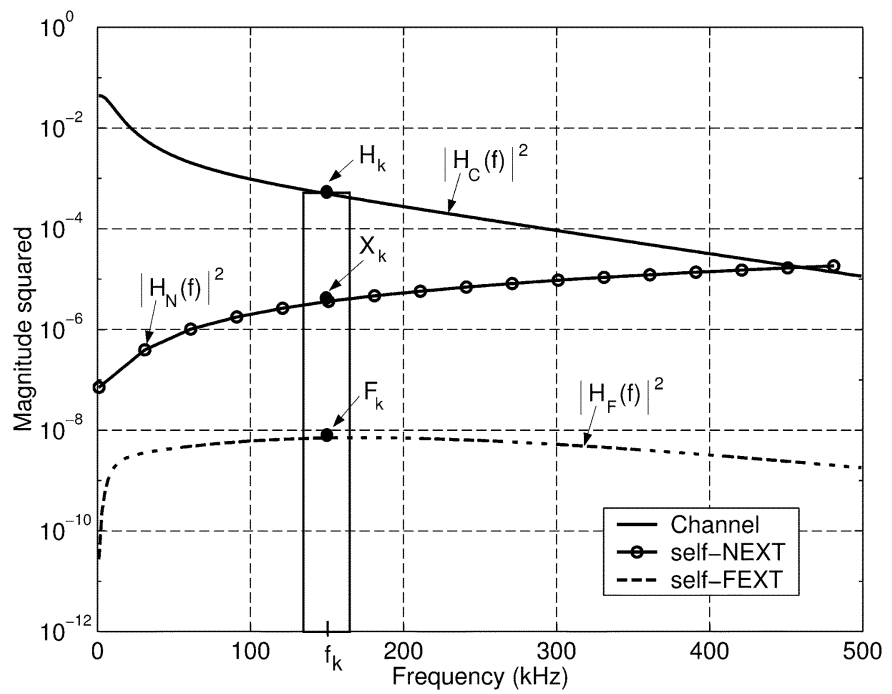

Fig. 1. Magnitude-squared transfer function of the channel, 39 self-NEXT interferers, and 39 self-FEXT interferers for carrier serving area (CSA) loop 6 . The figure also shows a narrow frequency bin centered around frequency $f_{k}$ with the channel $\left(H_{k}\right)$, self-NEXT $\left(X_{k}\right)$, and self-FEXT $\left(F_{k}\right)$ transfer functions approximated by constants over the bin.

single frequency bin. A no-division signaling (NDS) ${ }^{1}$ scheme in frequency bin $k$ is one for which $s^{u}(f)=s^{d}(f) \neq 0$ for all $f$ in the bin; that is, both upstream and downstream transmissions occupy the band $\left|f-f_{k}\right| \leq(W / 2)$. A frequency-division signaling (FDS) scheme in bin $k$ is one for which $s^{u}(f)=0$ when $s^{d}(f) \neq 0$ for all $f$ in the bin and vice versa; that is, $s^{u}(f)$ and $s^{d}(f)$ are orthogonal within $\left|f-f_{k}\right| \leq(W / 2)$ (see Fig. 2).

\section{Optimized Signaling TeChNiQues}

Given the channel, noise, and crosstalk characteristics of the DSL cable, we maximize the overall capacity of all $\mathcal{S}$-service lines by choosing between NDS and FDS in each frequency bin.

\section{A. Optimal Discrete Transmit Spectra}

In the presence of self-interference, our approach is similar to that in [2]. We can easily derive test conditions [3] to determine the signaling nature (FDS or NDS) in bin $k$

$$
\begin{aligned}
& \text { If } X_{k}^{2}-F_{k}^{2}-H_{k} F_{k}<0 \text {, then }
\end{aligned}
$$

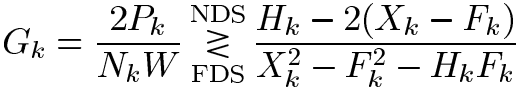

$$
\begin{aligned}
& \text { else if } X_{k}^{2}-F_{k}^{2}-H_{k} F_{k}>0 \text {, then } \\
& G_{k}=\frac{2 P_{k}}{N_{k} W} \underset{\text { FDS }}{\stackrel{N D S}{\gtrless}} \frac{H_{k}-2\left(X_{k}-F_{k}\right)}{X_{k}^{2}-F_{k}^{2}-H_{k} F_{k}} .
\end{aligned}
$$

${ }^{1}$ NDS was referred to as EQPSD signaling in [2] and [7]-[10]. 

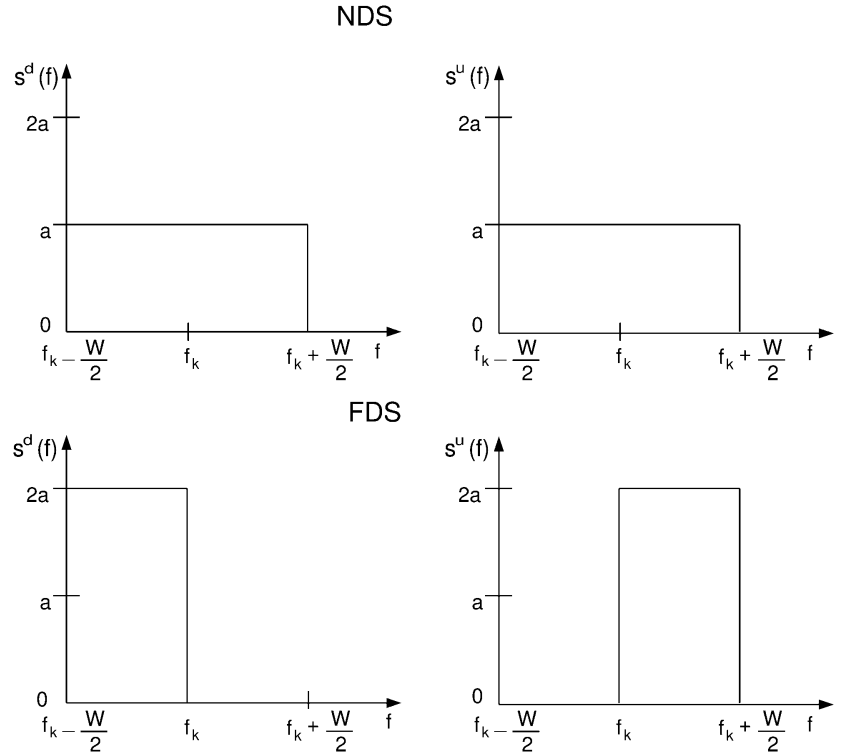

Fig. 2. Transmit spectra for NDS and FDS schemes in a single frequency bin $k$ (see Fig. 1). In the FDS scheme, the spectra $s^{u}(f)$ and $s^{d}(f)$ share disjoint frequency bands and are orthogonal to each other. This eliminates self-NEXT, but reduces bandwidth (and capacity) by half.

The test conditions are physically reasonable: use FDS in the bin if the signal-to-noise ratio (SNR) $\left(G_{k}\right)$ is low, compared with the self-interference transfer functions $\left(X_{k}, F_{k}\right)$; otherwise, use NDS.

In the absence of noise $\left(N_{k}=0\right)$, we can derive the signaling test condition

$$
X_{k}^{2}-F_{k}^{2}-H_{k} F_{k} \underset{\text { FDS }}{\stackrel{\mathrm{NDS}}{\gtrless} 0 .}
$$

To obtain the complete discrete optimal spectra, we apply the test conditions (1) and (2) to each frequency bin in $[0, B]$. To distribute the total power over the NDS and FDS regions, we employ a variation of waterfilling [1]. A simple iterative algorithm yields the complete discrete optimal transmit spectra [2] (see Fig. 3).

\section{B. Optimal Grouping of Bins}

Discrete transmit spectra must use discrete multitone (DMT) for modulation. A scheme like contiguous spectra with a reduced number of transitions are desirable for implementation using broadband modulation schemes such as carrierless amplitude/phase modulation (CAP) or quadrature amplitude modulation (QAM) [5]. We now present a scheme to group FDS bins that yields contiguous spectra that achieve equal performance margins and equal upstream and downstream average powers (see Fig. 4). Let $E_{\mathrm{FDS}}$ denote the FDS spectral region and $E_{\mathrm{NDS}}$ the NDS spectral region. A simple iterative algorithm yields the optimal grouping of bins.

1) Solve for the discrete optimal spectrum $S^{u}(f)$ according to the algorithm in Section II-A.

2) Obtain $S^{d}(f)$ from $S^{u}(f)$ by symmetry, i.e., $S^{d}(f)=$ $S^{u}(f) \forall f$ in $E_{\mathrm{NDS}}$ and $S^{d}(f)$ orthogonal to $S^{u}(f) \forall f$ in $E_{\mathrm{FDS}}$. Merge $S^{d}(f)$ and $S^{u}(f)$ to form $S(f)$ as

$$
S(f)= \begin{cases}S^{u}(f)=S^{d}(f) & \forall f \in E_{\mathrm{NDS}} \\ S^{u}(f)+S^{d}(f) & \forall f \in E_{\mathrm{FDS}} .\end{cases}
$$
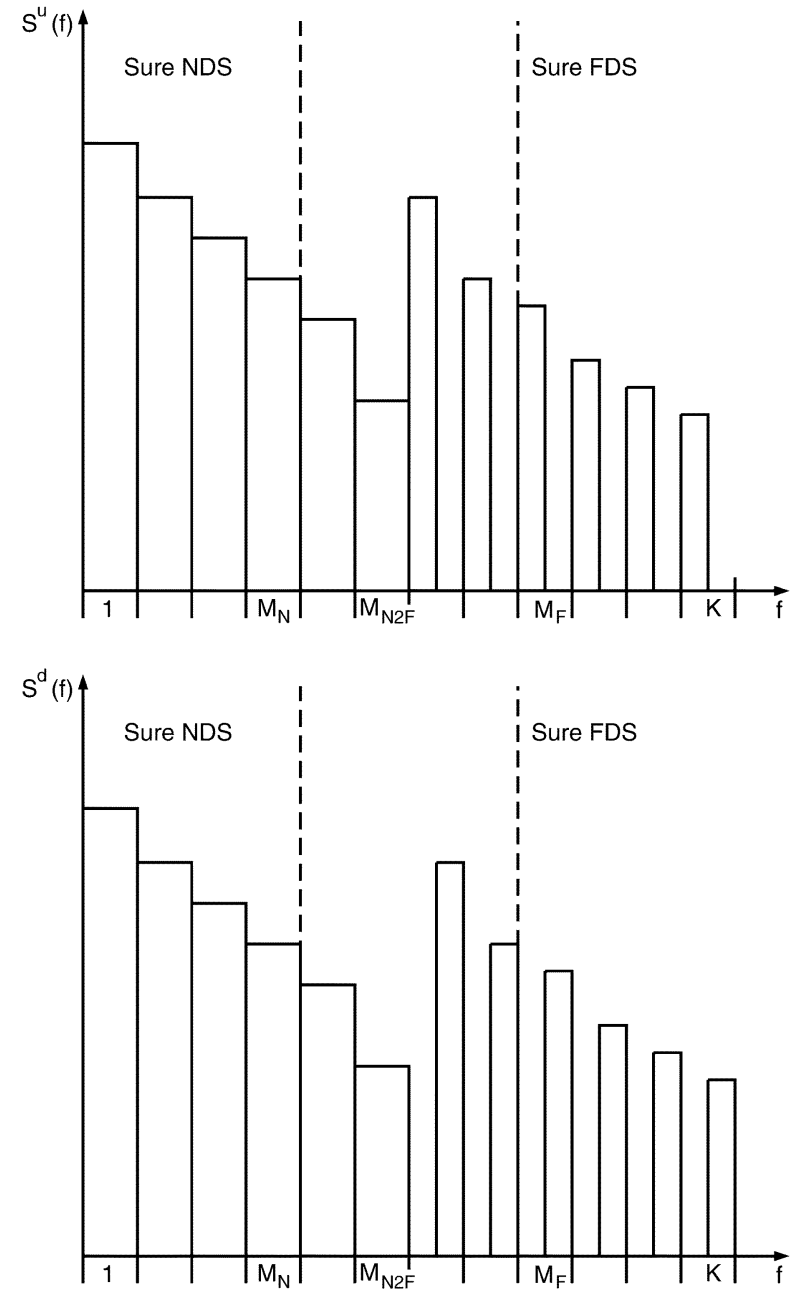

Fig. 3. Upstream and downstream transmit spectra showing regions employing NDS and FDS. The bins $\left[1, M_{\mathrm{N}}\right]$ employ NDS and the bins $\left[M_{\mathrm{F}}, K\right]$ employ FDS, as determined by evaluating test conditions (1) and (2) in each bin. The bin $M_{\mathrm{N} 2 \mathrm{~F}}$ is the optimal NDS-to-FDS switchover bin. Bins $\left[1, M_{\mathrm{N} 2 \mathrm{~F}}\right]$ employ NDS, and bins $\left[M_{\mathrm{N} 2 \mathrm{~F}}+1, K\right]$ employ FDS.

3) Choose a bin $M_{C} \in\left(M_{\mathrm{N} 2 \mathrm{~F}}, K\right]$, where $M_{\mathrm{N} 2 \mathrm{~F}}$ is the optimal NDS-to-FDS switchover bin. Estimate bin $M_{G} \in$ $\left(M_{C}, K\right]$ such that we have equal average powers in both directions. Group the bins of $S(f)$ to obtain upstream and downstream transmit spectra

$$
\begin{aligned}
& S_{\text {opt }}^{u}(f)= \begin{cases}S(f) & \forall f \text { in } E_{\mathrm{NDS}} \\
& \forall f \text { in bins }\left(M_{C}, M_{G}\right] \\
0, & \text { otherwise }\end{cases} \\
& S_{\text {opt }}^{d}(f)= \begin{cases}S(f) & \forall f \text { in } E_{\mathrm{NDS}} \\
& \forall f \text { in bins }\left(M_{\mathrm{N} 2 \mathrm{~F}}, M_{C}\right] \\
& \forall f \text { in bins }\left(M_{G}, K\right] \\
0, & \text { otherwise. }\end{cases}
\end{aligned}
$$

4) Iterate Step 3 for different choices of $M_{C}$ and $M_{G}$. Choose the final values for bins $M_{C}$ and $M_{G}$ to achieve equal performance margins and equal average powers in both directions of transmission.

Since we have two degrees of freedom (bin choices $M_{C}$ and $M_{G}$ ) to obtain equal margins and equal average powers, the algorithm is guaranteed to converge [3]. 

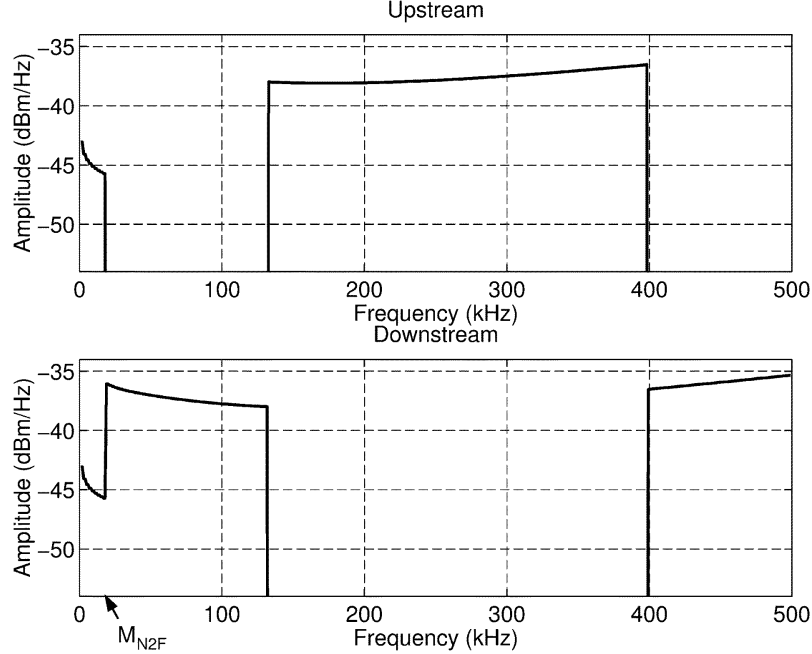

(a)
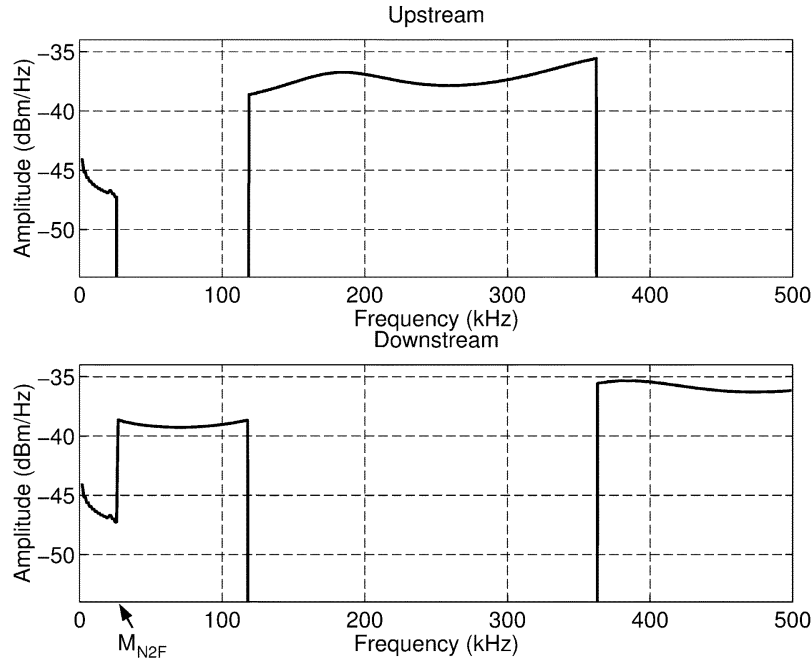

(b)

Fig. 4. Optimal upstream $\left(S^{u}(f)\right)$ and downstream $\left(S^{d}(f)\right)$ transmit spectra for HDSL2. (a) On CSA loop 6. (b) On CSA loop 4 (having bridged taps) with 39 self-NEXT and 39 self-FEXT interferers and AGN of $-140 \mathrm{dBm} / \mathrm{Hz}$. NDS takes place to the left of switchover frequency $M_{\mathrm{N} 2 \mathrm{~F}}$, and FDS to the right.

\section{Optimization Under a Frequency-Domain Peak-Power Constraint}

The need to limit the transmit spectrum could arise due to a standardized fixed PSD mask, or from spectral compatibility considerations. Here, we impose a frequency-domain peak power constraint, in addition to the average power constraint. We obtain optimal transmit spectra for an $\mathcal{S}$ service under these constraints in the absence of self-interference (refer to [3] for the solution in the presence of self-interference).

The problem statement is similar to that in [11], but without the equal-capacity constraint and with the additional frequency-domain peak-power constraint. Consider a line carrying a service $\mathcal{S}$ in the presence of different service interference and channel noise. The twisted-pair channel can be treated as a Gaussian channel with colored Gaussian noise [6]. The channel capacities (in bits per second) along with the average-power constraints are given by [2], [3], and [11]. In addition, we have new peak-power constraints

$$
S^{u}(f) \leq Q^{u}(f) \forall f, \quad S^{d}(f) \leq Q^{d}(f) \forall f .
$$

The average-power and peak-power constraints are linear and differentiable. Further, the capacity objective function is concave [12]. Any solution to this nonlinear optimization problem must therefore satisfy the Karush-Kuhn-Tucker (KKT) necessary conditions for optimality [13]. For a concave objective function and linear, differentiable constraints, any solution that satisfies the KKT necessary conditions is a unique globally optimal solution [13]. Thus, we seek any solution that satisfies these conditions.

"Peak-constrained waterfilling"2 yields the optimal solution to [11] and (7) [3]. The optimal transmit spectrum is given by

$$
S_{\text {opt }}^{u}(f)= \begin{cases}\lambda-\frac{N(f)}{\left|H_{C}(f)\right|^{2}}, & f \in E_{\mathrm{pos}} \\ Q^{u}(f), & f \in E_{\max } \\ 0, & \text { otherwise }\end{cases}
$$

with $\lambda$ a Lagrange multiplier. The spectral regions $E_{\text {pos }}$ and $E_{\max }$ are specified by

$$
\begin{aligned}
E_{\text {pos }} & =\left\{f: 0 \leq S^{u}(f) \leq Q^{u}(f)\right\} \\
E_{\max } & =\left\{f: S^{u}(f)>Q^{u}(f)\right\} .
\end{aligned}
$$

We vary the value of $\lambda$ to achieve the optimal transmit spectrum $S_{\mathrm{opt}}^{u}(f)$ that satisfies the average- and peak-power constraints. It is easily shown that this solution satisfies the KKT conditions [3].

\section{SimUlation RESUlTS}

We consider HDSL2, a $1.544 \mathrm{Mb} / \mathrm{s}$ symmetric bit-rate service, for our simulations [14]-[16]. Fig. 4 illustrates the optimal transmit spectra for the standard test case CSA loop 6 [5] for HDSL2 in the presence of 39 HDSL2 self-NEXT and self-FEXT interferers. ${ }^{3}$ High self-NEXT at high frequencies forces the optimal upstream and downstream spectra to separate in frequency, giving rise to an FDS region.

The performance margin of a channel measures the maximum SNR degradation that a channel can sustain before being unable to transmit at a particular bit rate and BER [20]. Table I lists the uncoded performance margins of the optimal transmit spectra versus those obtained using the OPTIS transmit spectra. ${ }^{4}$ For HDSL and T1 interferers, only the NEXT powers were considered; for HDSL2, "self" comprises both self-NEXT and self-FEXT. Equal performance margins were obtained for upstream and downstream optimal transmit spectra. In all cases, the optimal scheme significantly outperforms OPTIS (which uses fixed transmit spectra), with performance gains up to $16 \mathrm{~dB}$.

${ }^{2}$ Peak-constrained waterfilling can be likened to filling water in a closed vessel with uneven top and bottom surfaces.

${ }^{3}$ Simulation details: Bit rate fixed at $1.552 \mathrm{Mb} / \mathrm{s}$. Total average input power in each direction $P_{\max }=19.78 \mathrm{dBm}$. Different service interference models obtained from [17, Annex B of T1.413-1995], the ADSL standard, with exceptions as in [15]. Self-NEXT interference modeled as a two-piece Unger model [18]. Margins calculated according to [19]. OPTIS (ANSI T1E1.4's HDSL2 standard for transmit PSD masks) transmit spectra obtained by tracking $1 \mathrm{dBm} / \mathrm{Hz}$ below the OPTIS PSD masks [16]. OPTIS performance margin figures from [16]. AGN of $-140 \mathrm{dBm} / \mathrm{Hz}$ added to the interference. DMT scheme: Sampling frequency $f_{s}=1000 \mathrm{kHz}$. Bin width $W=2 \mathrm{kHz}$. Start frequency $=1 \mathrm{kHz}$. Bit-error rate $(\mathrm{BER})=10^{-7}$. SNR gap $=9.8 \mathrm{~dB}$. No cyclic prefix. No limitation on maximum number of bits per tone. See [20] for more details.

${ }^{4}$ OPTIS numbers were obtained from [16]. Diff $=$ Difference between worst-case optimal and worst-case OPTIS 
TABLE I

Uncoded (No Coding Gain) Performance Margins (IN DECIBELS) FOR HDSL2 ON CSA LOOP 6: OPTIS [16] VERSUS OPTIMAL

\begin{tabular}{l||l|c|c|c|c|c}
\hline \multirow{2}{*}{ Crosstalk source } & & \multicolumn{2}{|c|}{ OPTIS } & \multicolumn{2}{c|}{ Optimal } & \multirow{2}{*}{} \\
\cline { 3 - 7 } & DSL service & Up & Dn & Up & Dn & Diff \\
\hline \hline 49 HDSL & HDSL2 & 2.7 & 12.2 & 18.5 & 18.5 & $\mathbf{1 5 . 8}$ \\
\hline $25 \mathrm{~T} 1$ & HDSL2 & 19.9 & 17.5 & 21.3 & 21.3 & $\mathbf{3 . 8}$ \\
\hline 39 self & HDSL2 & 2.1 & 9.0 & 18.3 & 18.3 & $\mathbf{1 6 . 2}$ \\
\hline 24 self +24 T1 & HDSL2 & 4.3 & 1.7 & 5.4 & 5.4 & $\mathbf{3 . 7}$ \\
\hline
\end{tabular}

\section{CONCLUSIONS}

Optimal transmit spectra adapt to surrounding interference and yield large performance-margin gains (up to $16 \mathrm{~dB}$ ), compared with current fixed-spectra schemes for DSL communication. We can trade these increased performance margins for increased bit rates or decreased average transmit power. Optimal spectra are not bound to any particular modulation scheme. Vendor advantage can be achieved by adapting spectra underneath fixed, standardized PSD masks. By design, optimal transmit spectra achieve good spectral compatibility with existing services [3]. Our results assume some knowledge of the characteristics of the neighboring interfering services [3], but these are often known or can be estimated in practice.

\section{REFERENCES}

[1] J. Aslanis and J. Cioffi, "Achievable information rates on digital subscriber loops: Limiting information rates with crosstalk noise," IEEE Trans. Commun., vol. 40, pp. 361-364, Feb. 1992.

[2] A. Sendonaris, V. Veeravalli, and B. Aazhang, "Joint signaling strategies for approaching the capacity of twisted pair channels," IEEE Trans. Commun., vol. 46, pp. 673-684, May 1998.
[3] R. Gaikwad and R. Baraniuk. (1998) Spectral optimization and joint signaling techniques for communication in the presence of crosstalk. Rice Univ., Elect. and Comput. Eng. Dept., Tech. Rep. 9806, Houston, TX. [Online]. Available: http://www.dsp.rice.edu/publications

[4] J. Werner, "The HDSL environment," IEEE J. Select. Areas Commun., vol. 9, pp. 785-800, Aug. 1991.

[5] W. Chen, DSL Simulation Techniques and Standards Development for Digital Subscriber Line Systems. New York: Macmillan, 1998.

[6] K. Kerpez, "Near-end crosstalk is almost Gaussian," IEEE Trans. Commun., vol. 41, pp. 670-672, Jan. 1993.

[7] R. Gaikwad and R. Baraniuk, "Optimal transmit spectra for communication in the presence of crosstalk," in Proc. IEEE Int. Conf. Communications, June 1999.

[8] _ "Optimal transmit spectra for HDSL2," ANSI, T1E1 Contrib. T1E1.4/98-162R1, 1998.

[9] —_, "Optimal transmit spectra for HDSL2 under a peak frequency-domain power constraint," ANSI, T1E1 Contrib. T1E1.4/98-188R2, 1998.

[10] — , "Transmit spectra adaptive to noise and interference at activation for HDSL2,” ANSI, T1E1 Contrib. T1E1.4/98-258, 1998.

[11] R. Gallager, Information Theory and Reliable Communication. New York: Wiley, 1968.

[12] T. Cover and J. Thomas, Elements of Information Theory. New York: Wiley-Interscience, 1991.

[13] R. Horst, P. Pardalos, and N. Thoai, Introduction to Global Optimization. Norwell, MA: Kluwer, 1995.

[14] M. Rude, M. Sorbara, H. Takatori, and G. Zimmerman, "A proposal for HDSL2 transmission: OPTIS,” ANSI, T1E1 Contrib. T1E1.4/97-238, 1997.

[15] G. Zimmerman, "Performance and spectral compatibility of OPTIS HDSL2," ANSI, T1E1 Contrib. T1E1.4/97-237, 1997.

[16] J. Girardeau, M. Rude, H. Takatori, and G. Zimmerman, "Updated OPTIS PSD mask and power specification for HDSL2," ANSI, T1E1 Contrib. T1E1.4/97-435, 1997.

[17] Network and customer installation interfaces: Asymmetric Digital Subscriber Line (ADSL) Metallic Interface, American Nat. Standards Inst. (ANSI), T1E1 Standard T1.413-1995, 1995.

[18] K. Kerpez, "Full-duplex 2B1Q single-pair HDSL performance and spectral compatibility," ANSI, T1E1 Contrib. T1E1.4/95-127, 1995.

[19] G. Zimmerman, "Normative text for spectral compatibility evaluations," ANSI, T1E1 Contrib. T1E1.4/97-180R1, 1997.

[20] M. Barton and M. Honig, "Optimization of discrete multitone to maintain spectrum compatibility with other transmission systems on twisted copper pairs," IEEE J. Select. Areas Commun., vol. 13, pp. 1558-1563, Dec. 1995. 